\title{
Network Calibration interval optimization algorithm based on NLGA-GM $(1,1)$
}

\author{
Xinchen Cui, Zhenlin Chen \\ Department of Engineer Weapon Science and Technology, Naval Aeronautical and Astronautical \\ University, Yantai 264001, P.R.China \\ cuixinchen@sina.com
}

Keywords: Genetic algorithm, gray model, nonlinear programming, calibration interval.

Abstract. In order to optimize the calibration intervals, predict its calibration data using gray GM (1, 1) model. For lack of modeling parameter vector solving method, use genetic algorithms to seek optimal parameter vector; use nonlinear programming function's good ability of local searching to improve GA; finally, improve the initial base value selection of GM $(1,1)$ model. NLGA-GM $(1,1)$ forecasting model is given to optimize the calibration interval, and the optimizing model is validated through experiments. The results show that the improved model is better than the traditional GM at structure, data fitting accurateness and the calibration interval forecasts requirements.

\section{Introduction}

Calibration interval of measuring instrument is a multifactor integrated time data impacted by production technology, service environment and frequency, staffing levels and other confounding factors, it often exits bias. But in the actual calibration, the calibration interval is usually recommended by the manufacturer according to other similar instrument status. Therefore it is necessary in practice, based on historical data of calibration parameters to optimize the calibration of measuring instruments interval.

In the study of the calibration interval optimization algorithms, because of the good prediction results for small sample nonlinear data, gray model received widespread attention, and the application of GM $(1,1)$ model is more frequent. Many documents use $\operatorname{GM}(1,1)$ model to predict the calibration parameter directly [1], the prediction effect of this approach is not very satisfactory. For GM $(1,1)$ model transformation, document [2] proposed GM $(1,1)$ power model, but this model is not suitable for predicting the calibration data which is stable and has certain trends. Document [3] proposed the idea of residuals processing. These methods are further data proceeding after GM $(1,1)$ model prediction, but they can't improve the model itself according to the characteristics of the calibration data and predictive modeling mechanism.

In this paper, the combining method of genetic algorithms and nonlinear programming is used to solve the function of $\operatorname{GM}(1,1)$ model parameter vector optimally; at the same time based on real-time requirements of calibration data prediction, $\operatorname{GM}(1,1)$ prediction algorithms initial iteration base value has been improved, the prediction model is more in line with the requirements of the calibration interval forecasts. Examples were used to analyze and verify.

\section{Solve parameters of GM $(1,1)$ prediction algorithm}

The white differential equation of $\operatorname{GM}(1,1)$ model is given as follow:

$$
\frac{d x^{(1)}}{d t}+a x^{(1)}=b
$$

When solving the white differential equation, the solution is set as follows:

$$
x^{(1)}(t)=C e^{-a t}+\frac{b}{a}
$$

When $t=1$ :

$$
x^{(1)}(1)=x^{(0)}(1)
$$




$$
C=\left[x^{(0)}(1)-\frac{b}{a}\right] e^{-a}
$$

The predictive value GM $(1,1)$ model is resulted as follows:

$$
\hat{x}^{(0)}(k)=\left(1-e^{a}\right)\left(x^{(0)}(1)-\frac{b}{a}\right) e^{-a(k-1)}
$$

Further, according to the principles of $\operatorname{GM}(1,1)$ model, the parameter vector in equation $(5)$ is solving based on the least squares method. Therefore, use the genetic algorithm to obtain optimal parameters so that the prediction accuracy can be improved.

\section{NLGA-GM $(1,1)$ prediction model}

Genetic Algorithm. Genetic Algorithm is to learn the theory of evolution and natural selection principles of genetics and genetic mechanisms, derived from a random search algorithm optimization. GA introduced concept of populations, genes and chromosomes into algorithm. At first, genes are combined to produce the first generation population. Then according to the "survival of the fittest" evolutionary thinking, evolve from generation to generation, resulting in more optimal solution.

Individual selection of each generation, the main principle is to adapt to the size of the "survival" bigger chance to participate in a combination of genetic operators' crossover and mutation to generate new populations, namely the issue of new solutions of greater fitness. As this process of natural evolution, the offspring population is stronger than the previous generation of adaptive population, by the best individual to decode the last population; you can get an approximate optimal solution.

Nonlinear Programming function optimizing Genetic Algorithm. As a new search algorithm, genetic algorithm is different from traditional search algorithms, with its simple and universal provide better stability for solving nonlinear programming problems new framework. But there are also disadvantages of genetic algorithms, because the use of selection, crossover and mutation operators to optimize the search, a strong global search capability, but its local search capability is weak, prone to premature convergence, which will remain in a local maximum optimal solution suboptimal value, usually only get the question, is not guaranteed to converge to the optimal solution.

To solve this problem, based on the nonlinear programming function good ability of local searching, improve the genetic algorithm.

The normal nonlinear programming algorithm steps are as follows:

i) Given the initial point $x^{(0)}$, the initial matrix $H_{0}=I_{n}$, setting accuracy $\varepsilon>0$.

ii) If the fitness function at this point of the partial derivative, then stop the algorithm $\left\|\nabla f\left(x^{(0)}\right)\right\| \leq \varepsilon$, the minimum point $x^{(0)}$; otherwise, go to step iii.

iii) Take the search direction

$p^{(0)}=-H_{0} \nabla f\left(x^{(0)}\right)$, at the same time to make $k=0$.

iv) $f\left(x^{(k)}+t_{k} p^{(k)}\right)=\min _{t \geq 0} f\left(x^{(k)}+t p^{(k)}\right)$

$$
x^{(k+1)}=x^{(k)}+t_{k} p^{(k)}
$$

v) If the fitness function partial derivatives of point $x^{(k+1)}$ is $\left\|\nabla f\left(x^{(k+1)}\right)\right\| \leq \varepsilon$, then stop the algorithm, minimal point is $x^{(k+1)}$; otherwise, go to step vi.

vi) If $k+1=n$, then make $x^{(0)}=x^{(n)}$, go to step iii; otherwise, go to step vii.

vii) The direction correction formula is given as follows:

$$
H_{k+1}=H_{k}+\frac{\left(x^{(k+1)}-x^{(k)}\right)\left(x^{(k+1)}-x^{(k)}\right)^{T}}{\left(x^{(k+1)}-x^{(k)}\right)^{T}\left(\nabla f\left(x^{(k+1)}\right)-\nabla f\left(x^{(k)}\right)\right)}-\frac{H_{k}\left(\nabla f\left(x^{(k+1)}\right)-\nabla f\left(x^{(k)}\right)\right)\left(\nabla f\left(x^{(k+1)}\right)-\nabla f\left(x^{(k)}\right)\right)^{T} H_{k}}{\left(\nabla f\left(x^{(k+1)}\right)-\nabla f\left(x^{(k)}\right)\right)^{T} H_{k}\left(\nabla f\left(x^{(k+1)}\right)-\nabla f\left(x^{(k)}\right)\right)}
$$

viii) $p^{(k)}=-H_{k+1} \nabla f\left(x^{(k+1)}\right)$

Then make $k=k+1$, and go to step iv.

General nonlinear programming algorithm optimization shows its basic idea that is based on the descent gradient, and according to the partial derivative of the value of each point and the value of the 
fitness function, in the process of searching the optimal solution constantly corrected search for direction in the vicinity of the second tentative point approximation, resulting in qualifying optimal solution. Because of this optimization approach nonlinear function, it has good local optimization capabilities, combined with GA optimization process, establish NLGA based optimization of GM $(1,1)$ model, to further improve the accuracy of the model and run speed.

Improvement of GM $(1,1)$ model iteration base value. According to equation (3), the initial base value of $\operatorname{GM}(1,1)$ is set $\hat{x}^{(0)}(1)=x^{(0)}(1)$, but the first data is the oldest information, the predictive value and the actual value must be greater deviations.

In response to these problems, based on real significance of calibration, set up:

$$
\hat{x}^{(0)}(n)=x^{(0)}(n)
$$

Initial conditions iteration by the formula (10) is set to re-calculate the $\mathrm{GM}(1,1)$ prediction equations obtained. The initial iteration of the base value for $\operatorname{GM}(1,1)$ prediction model was improved such that the expected sequence through the latest calibration points, through this process, so that the model can predict the future value of the time calibration data is more consistent with the law of development.

Modeling steps. Improved NLGA-GM $(1,1)$ model is established to search parameters solution, while the use of non-linear programming algorithm for local search, resulting in optimized predictive value, and modeling follows:

i) Enter the original calibration data sequence, and put the $\operatorname{GM}(1,1)$ parameter vector into the genetic algorithm. A random initial population is generated, and then set the size of the population, the biggest evolution algebra, fixed evolutionary factor, precision target and mutation probability.

ii) GA objective function is the predictive mean square error :

$Y_{n}=\frac{1}{n} \sum_{k=1}^{n}\left(x^{(0)}(k)-\hat{x}^{(0)}(k)\right)^{2}$, and the fitness function is $f\left(Y_{n}\right)=\frac{1}{Y_{n}}$, the greater value means the better forecast result, and the better parameters solution.

iii) Individual fitness value is calculated in line with the optimization criteria to judge whether or not, if met, will be the best individual and optimal output, then the end of the process; if not, then go to step iv.

iv) Choose renewable individual based on the roulette strategy, so $P P_{i}=\sum_{j=1}^{i} p_{i}, P P_{0}=0$, in it $P P_{i}$ is the cumulative probability, $p_{i}$ is the probability of individual choice, which is calculated as:

$$
p_{i}=\frac{f\left(Y_{n}\right)}{\sum_{i=1}^{N P} f\left(Y_{n}\right)}
$$

v) To determine whether the number is multiplying, if satisfied, the nonlinear optimization in Section 3.2 of the method to accelerate the evolutionary process, the fitness function takes $Y_{n}$, after the non-linear optimization, go to step 3; if not satisfied, go to Step iii.

\section{Simulation and Analysis of Results}

In this paper, the data of document [6] is used to simulate, digital millimeter E1412A monitoring Multi-Product Calibrator F5500A every month. Test data were shown in Table 1. 
Table 1 Measure Calibration Test Data \%

\begin{tabular}{ccc}
\hline SN & time & Uncertainty \\
\hline 1 & $2003-07$ & 0.0062 \\
2 & $2003-08$ & 0.0066 \\
3 & $2003-09$ & 0.0063 \\
4 & $2003-10$ & 0.0070 \\
5 & $2003-11$ & 0.0068 \\
6 & $2003-12$ & 0.0071 \\
7 & $2004-01$ & 0.0081 \\
8 & $2004-02$ & 0.0074 \\
9 & $2004-03$ & 0.0080 \\
10 & $2004-04$ & 0.0081 \\
11 & $2004-05$ & 0.0079 \\
12 & $2004-06$ & 0.0083 \\
\hline
\end{tabular}

GM $(1,1)$ Predictions. First, the calibration test data were conducted a feasibility test to verify that the model meets the requirements. Upon examination, all ratio of the data sequences fall within the coverage $\Theta=(0.8574,1.1663)$. After examination, calibration monitoring test data $\operatorname{GM}(1,1)$ model was established and solved. The experimental results were shown in Fig.1.

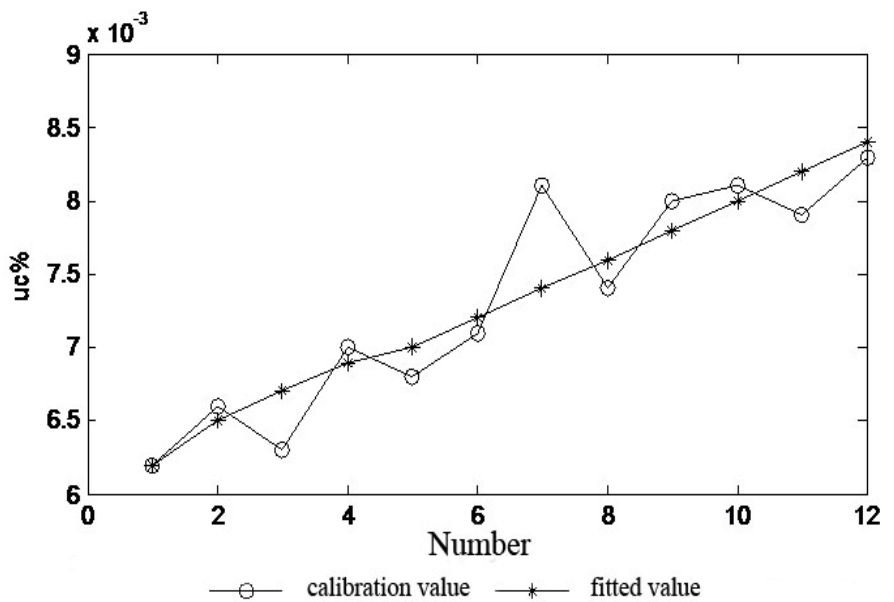

Fig.1 F5500A calibration data GM $(1,1)$ fitting results

NLGA-GM $(1,1)$ Predictions. Referring to the steps showed in 3.4 make modeling and data fitting. GA population size is popu $=50$, maximum evolution algebra is $G E N=100$, coding accuracy is eps $=1 e-10$, mutation probability is 0.1 , the crossover probability constant $k_{1}=0.5$ and $k_{2}=0.9$, fixed evolutionary factor is $N=5$. Fitting results showed in Figure 2.

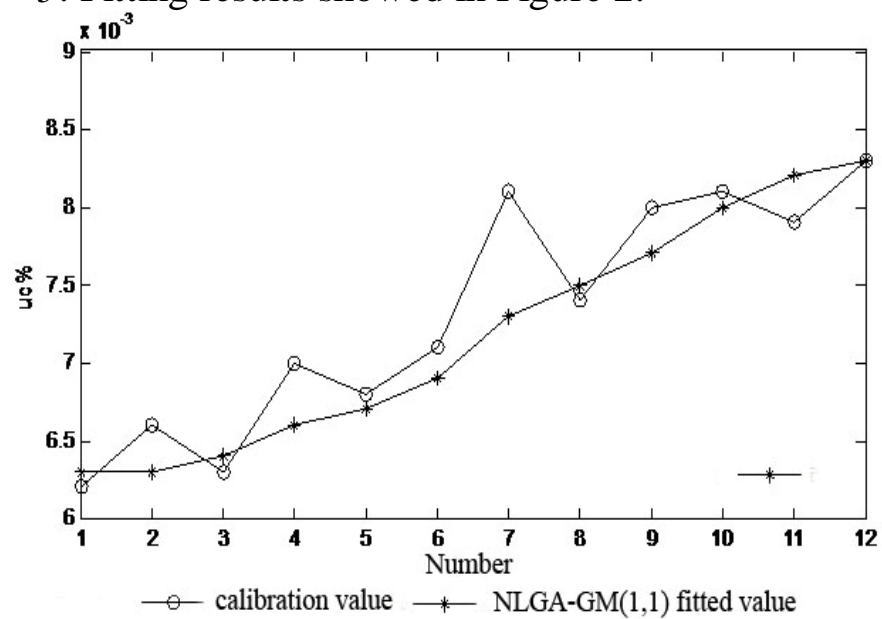

Fig. 2 NLGA-GM $(1,1)$ model F5500A data fitting

Contrast can be seen from Figure 1 and Figure 2; model predictions based NLGA optimization GM $(1,1)$ is closer to the actual measurements. 
Contrast of both models data fit accuracy is shown in Table 2.

By analyzing the data in Table 2 and Fig.1, by using genetic algorithm to optimize parameter vector GM $(1,1)$ model and improve its local search ability, the data fitting accuracy has been improved contrasted with traditional GM $(1,1)$ model.

Table 2 contrast of F5500A calibration data fitting MSE

\begin{tabular}{cc}
\hline Model & MSE \\
\hline Traditional GM $(1,1)$ & $7.5833 \mathrm{e}-0$ \\
NLGA optimization & $1.3000 \mathrm{e}-0$ \\
\hline
\end{tabular}

\section{Conclusions}

In this paper, the genetic algorithm is used to optimize the parameter vectors of GM $(1,1)$ model, while nonlinear programming function is used to make supplement and improvement the genetic algorithm global searching, and the iterative initial value is improved. Finally, the NLGA-GM $(1,1)$ prediction algorithm is given.

Make calibration data fitting and simulation results compared with improved model. Fitting results show that the improved predictive model has better structure , better data fitting accuracy and more consistent with the requirements of the calibration interval forecasts than the traditional $\mathrm{GM}(1,1)$ model.

This paper based on the modeling mechanism; improve the model's own structure principle. From the improved model data model fitting results, $\operatorname{GM}(1,1)$ model can predict trends data well. But at the same time we can see, the optimization of the parameters of the algorithm improvements, can't change the principles of trend forecasting model, and can't make a good fit for the fitted values of random fluctuations in the data.

\section{References}

[1] Li D C, Yeh C W, Chang C J. An improved grey-based approach for early manufacturing data forecasting [J]. Computers \& Industrial Engineering, 2009, 57(4):1161- 1167.

[2] Wang C H, Hsu L C. Using genetic algorithms grey theory to forecast high technology industrial output [J]. Applied Mathematics and Computation, 2008, 195(1): 256-263.

[3] Lin X, Shalom Y, Kirubarajan T. Exact multi-sensor dynamic bias estimation with local tracks [J]. IEEE Trans, on Aerospace and Electronic Systems, 2004, 40(2):576-590.

[4] Wang L, Shen T Z, Zhao Y. An improved adaptive genetic algorithm [J]. Systems Engineering and Electronics.2002 : 24(5) : 75-78.

[5] Zhao N, Chen X. Genetic algorithm based on the number of table identification [J]. Technology and Life, 2011, 21:209-209.

[6] Zhao R X, Meng X F, Wang G H. Dynamic optimization interval based on Grey Markov measuring instrument calibration [J]. Metrology Journal, 2007, 28(2):184-187. 